\title{
Isolated tuberculous epididymo-orchitis: an unusual presentation of tuberculosis
}

\author{
Vishnu Prasad Shenoy ${ }^{1}$, Shashidhar Viswanath ${ }^{1}$, Annet D'Souza ${ }^{1}$, Indira Bairy ${ }^{1}$, Joseph \\ Thomas $^{2}$
}

Departments of Microbiology ${ }^{1}$ and Urology ${ }^{2}$, Kasturba Medical College Manipal, Manipal University, India

\begin{abstract}
Isolated epididymo-orchitis is an unusual presentation of tuberculosis. A case of bilateral epididymitis and right-sided orchitis with scrotal involvement in a 38-year-old male patient is presented. Strong clinical suspicion of tuberculous etiology was confirmed by appropriate investigations of epididymal biopsy. The patient improved clinically with antitubercular therapy.
\end{abstract}

Key words: epididymo-orchitis; genitourinary; tuberculosis

J Infect Dev Ctries 2012; 6(1):92-94.

(Received 23 June 2011 - Accepted 01 December 2011)

Copyright () 2012 Shenoy et al. This is an open-access article distributed under the Creative Commons Attribution License, which permits unrestricted use, distribution, and reproduction in any medium, provided the original work is properly cited.

\section{Introduction}

Genitourinary tuberculosis (GUTB) is the second most common form of extrapulmonary tuberculosis after lymph node involvement [1]. It is estimated that GUTB comprises $30 \%$ of non pulmonary TB and can cause disease in the retroperitoneum, adrenal glands, kidney, urinary collecting system, male genital system, and female pelvic organs [2]. In approximately $28 \%$ of patients with GUTB, the involvement is solely genital [3]. In men, the sites most commonly affected are the epididymis followed by the prostate [4]; however; isolated epididymoorchitis may produce diagnostic difficulty while excluding a possible testicular neoplasm [3]. This report aims to present a case of isolated tuberculous epididymo-orchitis with no other tuberculous foci elsewhere in the body.

\section{Case report}

A 38-year-old previously healthy male from Kerala in southern India presented with complaints of bilateral scrotal swelling for four months. He gave a history of increased scrotal pain and discharging sinus for the past two months. He also provided a history of receiving medical treatment (details not provided) with which the swelling reduced partially. The patient was afebrile and did not complain of any urinary symptoms including frequent voiding, dysuria or hematuria. There was no history of back, flank or abdominal pain. There was no prior history of tuberculosis or therapy with anti-tubercular drugs. Past history was not significant and he led a married life and had two children.

Local examination revealed bilateral inguinal lymphadenopathy. External genitalia examination revealed normal meatus, enlarged right testis with thick cord, thickened left epididymis, and a sinus in the posterior scrotal wall. Direct per-rectal examination revealed Grade I non tender prostate. Systemic examination was normal. Routine hematological parameters were within normal limits. Scrotal ultrasonography showed bilateral epididymitis with granulomatous mass at the upper pole of the right testis, suggestive of chronic granulomatous inflammation.

Tubercular etiology was suspected based on clinical presentation and scrotal ultrasonography findings and was investigated accordingly. Right epididymal biopsy was performed under general anaesthesia. Gross examination showed multiple foci of caseous material along with foci of haemorrhage. Histopathological examination of the biopsy material showed granulomas composed of dispersed epithelioid histiocytes, langhans giant cells, lymphocytes and plasma cells with large areas of caseous necrosis. These features were consistent with tuberculosis. Fluorescent microscopy of the biopsy sample was negative for acid-fast bacilli. There was 
no pulmonary evidence of tuberculosis on the chest radiograph. Nested PCR (Bangalore Genei, Bangalore, India) for $M$. tuberculosis targeting insertion element IS6110 gave a negative result. Urine culture was sterile and the levels of tumor markers--beta-HCG $(<0.1 \mathrm{mIU} / \mathrm{ml})$ and AFP $(1.4$ $\mathrm{ng} / \mathrm{ml}$ ) — were within normal limits. A diagnosis of tuberculous epididymo-orchitis was made based on clinical suspicion and histopathology findings. Absence of renal involvement was indicated by lack of suggesting symptoms and fluorescent microscopic examination of urine was negative for acid-fast bacilli. Later $M$. tuberculosis was grown on Lowenstein-Jensen medium from the epididymal biopsy sample after eight weeks. The culture growth was confirmed by Accuprobe (Gen Probe, San Diego, USA) and by nested PCR (IS 6110).

The patient was treated with anti-tubercular drugs including rifampicin $(450 \mathrm{mg})$, isoniazid $(300 \mathrm{mg}$ ), ethambutol $(800 \mathrm{mg})$ and pyrazinamide $(1500 \mathrm{mg})$ for two months followed by a continuous phase with rifampicin (450 mg) and isoniazid (300 $\mathrm{mg}$ ) for four months. He improved clinically with reduction of local swelling and tenderness and had a clinical course of continuous improvement and antitubercular therapy was stopped at the end of six months.

\section{Discussion}

GUTB is still a major health problem in many developing countries including India. In India, the incidence of genital TB is nearly about 18\% [4]. Urogenital tuberculosis affects all age ranges, with predominance of males between the ages of 30 and 50 years [5]. Our patient was a 38-year-old male. Tuberculous foci in the epididymis are caused by metastatic spread of organisms through the bloodstream. Tuberculous epididymitis may be the first and only presenting symptom of GUTB [6]. Testicular involvement usually is the result of direct extension from the epididymis, and scrotal involvement suggests local extratesticular extension of the disease process [4].

Local symptoms of the disease are usually insidious and progressive, which can be confused with other infections, cysts and tumours [7]. Bilateral epididymal involvement and concomitant testicular lesion strongly suggest tubercular etiology, especially in patients with tubercular foci elsewhere in the body and failure to respond to conventional antibiotic therapy [4]. Male genital tuberculosis usually is associated with renal tuberculosis in $60 \%$ to $65 \%$ of cases or with pulmonary tuberculosis in approximately $34 \%$ of cases [5]. No renal or pulmonary involvement was seen in our patient considering the lack of symptoms and negative findings on the chest radiograph and ultrasonography of the abdomen. However, intravenous urography for renal involvement and trans-rectal ultrasonography for prostate involvement were not performed in our patient. Characteristic signs on IV pyelogram and computerized tomography are described as useful in depicting GUTB [6]. These tests, if performed, could have definitely ruled out involvement of the renal tract.

A positive culture or histological analysis of biopsy specimens, possibly combined with PCR, is required for a definitive diagnosis of GUTB [6]. However, the diagnosis of extrapulmonary tuberculosis is challenging for a number of reasons: the lack of adequate sample amounts or volumes; the apportioning of the sample for various diagnostic tests (histology/cytology, biochemical analysis, microbiology, and PCR), resulting in non uniform distribution of microorganisms; the paucibacillary nature of the specimens; and the presence of inhibitors that undermine the performance of nucleic acid amplification-based techniques [8]. The initial negative result by PCR could be explained by the above-mentioned reasons; however, considering the limitations of smear microscopy and culture, molecular amplification methods have a major role to play in diagnosing extra pulmonary tuberculosis. It has been noted that in $25 \%$ to $30 \%$ of cases, diagnosis of GUTB is established based on histological patterns and/or by detection of Mycobacterium tuberculosis complex by PCR [6]. Negi et al. performed a study on the diagnosis of extra pulmonary tuberculosis by PCR and noted that PCR has a potentially important role in strengthening the diagnosis of TB, especially in targeting IS6110 [9].

In diagnosing extrapulmonary tuberculosis, it is necessary to perform comprehensive evaluations, including histology, cytology and microbiological investigations (culture and PCR) and further clinical follow-up. A strong clinical suspicion of tuberculous etiology in the present case was followed by relevant histopathological and microbiological investigations, which led to confirmation of initial clinical diagnosis and the patient was successfully treated with anti tubercular drugs.

Furthermore, early and accurate diagnosis of GUTB is needed to avoid complications. Tuberculous 
epididymo-orchitis has considerable effects on fertility, as sperm count and motility may be reduced by blockage of the vas and/or secondary atrophy [4]. Our patient was advised to undergo semen analysis after completion of anti-tubercular therapy.

Tubercular epididymo-orchitis without renal involvement can be the sole presentation of GUTB, as seen in the present case. Hence tubercular etiology should be one of the differential diagnoses in patients presenting with scrotal swelling. Early diagnosis followed by multidrug chemotherapy will prevent any further complications on the functioning of the genito-urinary system.

\section{References}

1. Kapoor R, Ansari MS, Mandhani A, Gulia A (2008) Clinical presentation and diagnostic approach in cases of genitor urinary tuberculosis. Indian J Urol 24: 401-405.

2. Wise GJ and Marella VK (2003). Genitourinary manifestations of tuberculosis Urol Clin N Am 30: 111-121.

3. Shah H, Shah K, Dixit R, Shah KV (2004) Isolated Tuberculous epididymo-orchitis. Indian J Tubercl 51: 159162.

4. Das P, Ahuja A, Datta Gupta S (2008) Incidence, etiopathogenesis and pathological aspects of genitourinary tuberculosis in India: A journey revisited. Indian J Urol 24: 356-361.

5. Figueiredo AA, Lucon AM, Gomes CM, Srougi M (2008) Urogenital Tuberculosis: Patient classification in seven different groups according to clinical and radiological presentation. International Braz J Urol 34: 422-432.

6. Cek M, Lenk S, Naber KG, Bishop MC, Bjerklund Johansen TE, Botto H Grabe M, Lobel B, Redorta JP, Tenke P; Members of the Urinary Tract Infection (UTI) Working Group of the European Association of Urology (EAU) Guidelines Office (2005) EAU Guidelines for the Management of Genitourinary Tuberculosis. European Urology 48: 353-362.

7. Rajpal S, Dhingra VK, Malik M, Raj Kumar (2002) Tuberculous Epididymo-Orchitis Treated with Intermittent Therapy: A Case Report. Indian J Allergy Asthma Immunol 16: $51-54$.

8. Chakravorty S, Sen KM, Tyagi JS (2005) Diagnosis of Extrapulmonary Tuberculosis by Smear, Culture, and PCR Using Universal Sample Processing Technology. J Clin Microbiol 43: 4357-4362.

9. Negi SS, Anand R, Pasha ST, Gupta S, Basir SF, Khare S (2007) Diagnostic potential of IS6110, $38 \mathrm{kDa}, 65 \mathrm{kDa}$ and $85 \mathrm{~B}$ sequence- based polymerase chain reaction in the diagnosis of Mycobacterium in clinical samples. Indian J Med Microbiol 25: 43-49.

\section{Corresponding author}

Dr Vishnu Prasad Shenoy

Department of Microbiology

Kasturba Medical College

Manipal University

Manipal, India

Telephone: +91820-2922322

Email: vishnumanav@gmail.com)

Conflict of interests: No conflict of interests is declared. 